\title{
La traduction comme enquête anthropologique : esquisse d'une conception
}

Trying to conceive translation as an anthropological inquiry

\section{Carole Le Hénaff}

\section{(2) OpenEdition}

\section{Journals}

Édition électronique

URL : http://journals.openedition.org/educationdidactique/2460

DOI : 10.4000/educationdidactique.2460

ISSN : 2111-4838

Éditeur

Presses universitaires de Rennes

\section{Édition imprimée}

Date de publication : 30 mai 2016

Pagination : 49-66

ISBN : 978-2-7535-5060-5

ISSN : 1956-3485

Référence électronique

Carole Le Hénaff, "La traduction comme enquête anthropologique : esquisse d'une conception », Éducation et didactique [En ligne], 10-1 | 2016, mis en ligne le 30 mai 2018, consulté le 19 avril 2019. URL : http://journals.openedition.org/educationdidactique/2460 ; DOI : 10.4000/ educationdidactique. 2460 


\title{
LA TRADUCTION COMME ENQUÊTE ANTHROPOLOGIQUE: ESQUISSE D'UNE CONCEPTION
}

\author{
Carole Le Hénaff \\ ESPE de Bretagne, Laboratoire CREAD
}

\begin{abstract}
La traduction est un processus complexe à définir, qui peut prendre plusieurs formes, et qui est associé à des pratiques diverses. Afin d'en produire l'esquisse d'une conception, cet article analyse trois exemples « emblématiques » de la traduction, ainsi que les propos d'une traductrice avec laquelle nous avons mené un entretien autour de sa pratique. Notre étude, à la fois exploratoire et inaugurale pour de futures orientations de recherche, propose donc l'esquisse d'une certaine vision de la traduction, en tant qu'enquête anthropologique sur les langues et les cultures. La conception ainsi produite nous amène à réfléchir, en fin d'article, aux enjeux qu'une telle approche de la traduction pourrait impliquer dans l'enseignement des langues vivantes à l'école.
\end{abstract}

Mots-clés: traduction, enquête, anthropologie, didactique des langues, culture.

\section{Trying to conceive translation as an anthropological inquiry}

Defining the translation process is a complex task, because translating can take several forms, and is associated with diverse practices. In order to elaborate a first conception outline of this process, we analyze three "emblematic" examples of translation, combined with what a professional translator says about her practice. Thus, our study, which is both exploratory and opening paths for our future research, presents a sketch of a certain vision of what translation consists of, which is, an anthropological inquiry on languages and cultures. At the end of our paper, this conception leads us to reflect upon the implications that such an approach of translation could generate for the teaching of foreign languages.

Keywords: translation, inquiry, anthropology, didactics of foreign languages, culture.

Remerciements

À Luc Trouche, pour la diffusion de ses données.

À Jocelyne Bourbonnière, pour son regard d'experte sur sa pratique de traductrice. 


\section{INTRODUCTION}

Qu'est-ce que traduire? Il est difficile de répondre simplement à une telle question, tant les enjeux liés à ce processus sont divers et complexes. Depuis toujours, le concept de traduction a fait l'objet d'une certaine attention. Depuis quelques années, cette attention s'est accrue, en particulier au sein de l'Union Européenne et avec la mondialisation, et la traduction est devenue une forme incontournable de ce «fait social total» (Mauss, 1950) qu'est la communication.

Notre article ne se veut pas exhaustif sur la question de la traduction, maintes fois traitée depuis bien longtemps. Il s'agit davantage d'une conception de ce que peut être la traduction, au sens de l'étude d'un savoir savant (Chevallard, 1991) ainsi que d'une orientation pour notre propre travail en didactique des langues.

Cette étude est à la fois inaugurale et exploratoire, en particulier parce qu'elle constitue un point de départ à un axe de recherche plus général, sur les pratiques des traducteurs. Nous serons donc amenés, au fil de nos orientations de recherche, à préciser cette esquisse d'une certaine conception de la traduction, dont cet article représente le commencement.

L'objet de l'article est de proposer une conception de la traduction en interrogeant trois exemples emblématiques de cette pratique, et en nourrissant notre étude à l'aide des propos d'une " connaisseuse pratique » (Sensevy, 2011), c'est-à-dire une traductrice, avec laquelle nous avons mené un entretien. Parmi d'autres, Eco (2006) et Ladmiral (2010), en tant que traducteurs ayant théorisé leur pratique, ont souligné l'intérêt de la prise en compte de l'expérience des praticiens. Le but d'une telle démarche, qui est aussi l'objectif premier de l'article, est répondre à la question "qu'est-ce que traduire? », c'est-à-dire de décrire la substance de ce qui fait la pratique d'un expert dans ce domaine.

Les exemples choisis peuvent également être considérés comme des exemplaires (Kuhn, 1990) d'une manière d'envisager la pratique de la traduction à travers l'étude de ces énoncés dits « intraduisibles », qui sont en fait plutôt des énoncés qu'on «ne cesse jamais de traduire (Cassin, 2007), en particulier en ayant recours à plusieurs formes de descriptions. La question de l'intraduisible est récurrente lorsqu'on s'intéresse à la pratique de la traduction. Même si elle n'est pas toujours posée en tant que telle, c'est le plus souvent à l'appui d'exemples d'énoncés difficiles à transposer d'une langue à une autre, d'une culture à une autre, qu'on tente de répondre à " qu'est-ce que traduire?».

Ainsi, nous considérons les trois exemples choisis comme particulièrement emblématiques parce qu'ils donnent à voir comment, à travers plusieurs formes de descriptions, l'intraduisible prend des formes qu'on ne cesse de traduire. Malgré leur singularité, ces exemples réunissent beaucoup de caractéristiques propres aux enjeux de la pratique de la traduction, notamment la prise en considération essentielle des contextes culturels, historiques, politiques, etc., permettant de produire certaines formes d'équivalences entre les énoncés d'une langue à l'autre. Nous souhaitons donc, à travers l'étude de quelques points spécifiques liés à la traduction, produire une réflexion générale sur ses enjeux cruciaux. Les exemples emblématiques que nous avons choisis, croisés à l'analyse de notre entretien avec la traductrice, nous permettent d'illustrer une certaine conception de la traduction, en tant qu'enquête anthropologique.

Nous proposons, en fin d'article, quelques réflexions sur l'intégration de la traduction dans le domaine de l'enseignement des langues. Cette partie met notamment en lien les questionnements soulevés dans le cadre des recherches sur le plurilinguisme et l'Éveil aux Langues (entre autres, Candelier, 2003) avec la perspective adoptée dans notre article sur la question de traduction. Ces travaux ont contribué à faire émerger des approches de l'enseignement des langues développant la réflexion des élèves sur les systèmes linguistiques en contact les uns avec les autres, en particulier à travers des activités d'intercompréhension entre les langues, qui mettent en jeu une conception de la traduction différente de celle qui se pratique classiquement à l'école.

Les questions posées et les arguments développés dans notre texte sont issus de constats tirés d'un récent travail de thèse en didactique de l'anglais (Le Hénaff, 2013). Nous souhaitons ainsi produire les bases d'un arrière-plan conceptuel aux enjeux liés à cette pratique en classe de langue vivante, bien que cela ne constitue pas l'objectif premier de l'article, qui est avant tout de concevoir ce que peut signifier «traduire».

Notre article est structuré comme suit: en premier lieu, nous faisons le tour des principales grandes formes que peut prendre la traduction. Nous adoptons par la suite un point de vue historique afin 
de revenir sur quelques enjeux plus anciens liés à cette pratique. À la lumière de ce cadrage, nous nous interrogeons, en nous appuyant notamment sur notre entretien avec une traductrice, sur la pratique de la traduction. Nous abordons également cette pratique sous l'angle des institutions du sens (Descombes, 1996) et des conventions temporaires (Rastier, 2005) qui, de même que les variations individuelles, sociales et culturelles, façonnent les pratiques des traducteurs ainsi que la forme des traductions. Nous faisons appel à deux premiers exemples, celui du terme allemand d'origine freudienne, Trieb, puis celui de la traduction de " comédie » et " tragédie » par Averroès, qui donnent particulièrement à voir l'articulation de la langue avec la culture. Nous nous focalisons ensuite sur la question cruciale de l'intraduisible, et faisons usage d'un troisième exemple, la traduction du terme chinois 磨课, rapportée par Trouche (2015) suite à un séjour à Shanghai. La conception de la traduction ainsi esquissée nous amène à réfléchir aux implications didactiques qu'une autre approche de cette pratique, que celle actuellement en cours à l'école, pourrait engendrer. Nous revenons en conclusion à un point de vue plus général sur ce qu'est la traduction.

\section{QUELQUES ÉLÉMENTS DE CADRAGE}

\section{Les types de traduction}

"Traduction » est un terme très polysémique, qui peut caractériser énormément de pratiques, si on le prend dans le sens très vaste de conversion d'un langage dans un autre en vue de transmettre des informations. En allant très loin, on peut même avancer que " comprendre, c'est traduire " (Steiner, 1998). Selon Jakobson, il existe trois grandes formes de traduction:

1) Intralingual translation or rewording is an interpretation of verbal signs by means of other signs of the same language. 2) lnterlingual translation or translation proper is an interpretation of verbal signs by means of some other language. 3) Intersemiotic translation or transmutation is an interpretation of verbal signs by means of signs of nonverbal sign systems. (1959, p. 233.)

Ainsi, lorsqu'on parle de traduction, il peut s'agir, classiquement, d'équivalence d'énoncés entre des langues, mais aussi de changement de registre de langage, de vulgarisation scientifique, ou bien encore de représentation symbolique, ou schématisée. En didactique des langues également, on parle désormais plus souvent, non plus de traduction, mais de « médiation ${ }^{1} »$.

La traduction peut aussi être orale (le terme « interprétation » est généralement employé dans ce cas), elle peut être différée ou simultanée, officielle ou non. Un traducteur peut travailler sur des textes, ou bien à l'oral, ou encore sur des matériaux mêlant l'oral et l'image, comme au cinéma: dans ce dernier cas, lorsqu'il travaille sur le sous-titrage par exemple, il prend en compte un système sémiotique bien plus vaste qu'un texte écrit (images, sons, vitesse de lecture) afin de produire une traduction aisée à insérer dans les images. L'enjeu, lorsqu'on traduit un mot, un énoncé, un concept, est de rendre accessible à autrui une information (au sens le plus large) qu'il ne peut saisir avec ses seules connaissances langagières, culturelles, conceptuelles, etc. La conception de la traduction qui se dessine est la suivante: en lisant des signes puis en les racontant à l'aide d'autres signes, au sein d'un autre système, le traducteur est à la fois un déchiffreur de traces (Ginzburg, 1989) et un « raconteur », qui utilise un paradigme indiciaire (ibid.) lorsqu'il enquête sur le sens des mots. Car la traduction est une enquête (Dewey, 1938), avec pour point de départ une situation indéterminée. Cette situation indéterminée, cette incertitude, c'est la nouvelle forme d'équivalence que doivent prendre des mots dans une autre langue que celle dans laquelle ils ont été énoncés.

Lorsqu'on se demande " qu'est-ce que traduire? », cela peut donc recouvrir des pratiques très diverses; nous allons, pour notre part, nous centrer sur la question de la traduction d'une langue dans une autre, au sens de la recherche de formes d'équivalences entre deux (ou plusieurs) langues. En resserrant donc le champ d'observation à la traduction entre deux langues, on peut dire que traduire, c'est formuler dans deux langues différentes des énoncés plus ou moins équivalents, ou, du moins, produisant des effets ${ }^{2}$ plus ou moins équivalents sur les personnes à qui ces énoncés sont adressés. L'enjeu, lorsqu'on traduit, n'est donc pas tant de « coller » à une autre langue, à une autre culture, voire à une autre époque d'écriture, mais de faire fonctionner un système d'énoncés, de manière équivalente d'une langue à l'autre. 


\section{Aux origines de la traduction}

L'objectif de cette partie est de décrire, à la lumière de questions plus anciennes, ce que « traduction » a pu signifier au fil du temps. L'enjeu de ce détour par la mythologie et l'histoire est de pouvoir disposer d'un cadre historique afin d'en appréhender la pratique de manière plus nourrie.

Le mythe de la tour de Babel est régulièrement présenté comme le symbole, ou du moins une sorte de point de départ mythologique, de l'utopie de la langue unique face à l'immensité des besoins de traduction. Cette tour, dont Dieu aurait bloqué la construction en introduisant la multiplicité des langues afin que les hommes ne puissent plus s'entendre pour la construire et atteindre la divinité, est effectivement le symbole d'une question à la fois très ancienne et très actuelle, en résonance par exemple avec les enjeux de communication au sein de l'Union Européenne. Il y a quelques années, Eco (1993) a rappelé par ailleurs que « la langue de l'Europe, c'est la traduction $»$.

D'un autre côté, et pour demeurer encore un peu dans le passé, Cassin (2014a) explique que, dans la Grèce antique, la langue grecque, le logos, est considérée comme la seule véritable langue de la civilisation, les autres langages étant vus comme des barbarismes (« bar-bar » faisant référence à du « blabla » incompréhensible). Momigliano (1979) décrit ainsi les Grecs de l'époque comme un peuple « fièrement monolingue ». Par ailleurs, Cassin (2004) rappelle que le verbe grec «hellenizein » signifie à la fois:

- parler grec;

- parler correctement;

- helléniser (c'est-à-dire civiliser) un barbare;

- et plus tard dans l'Antiquité, exprimer en grec, soit traduire, comme par exemple la Bible, traduite au $\mathrm{II}^{\mathrm{e}}$ siècle.

Mais aucun verbe grec ne signifie réellement « traduire» (ibid.). La langue latine, par la suite, adaptera plus qu'elle ne traduira la langue et la culture grecques. La traduction mot à mot verbum pro verbo prendra par la suite son essor, avant que les traductions de la Bible par Saint Jérôme ne remettent en cause ce principe et rendent compte du sens tout en prenant des libertés avec le mot à mot (ibid.).

Cette question du logos, c'est-à-dire, ici, de la sacralisation d'une langue vue comme supérieure aux autres, a été aussi appliquée à la langue française au $\mathrm{XVIII}^{\mathrm{e}}$ siècle à travers les écrits de Rivarol, en particu- lier lors de son discours sur l'universalité de la langue française en 1784 au cours duquel il déclarait que:

«Dégagée de tous les protocoles que la bassesse invente pour la vanité et le pouvoir, elle en est plus faite pour la conversation, lien des hommes et charme de tous les âges, et puisqu'il faut le dire, elle est de toutes les langues la seule qui ait une probité attachée à son génie. Sûre, sociale, raisonnable, ce n'est plus la langue française, c'est la langue humaine. » (p. 20.)

Cassin, en référence à Rivarol, fait également le lien avec la lingua franca actuelle, le « Globish » (2014b), cette forme universelle et appauvrie de l'anglais qui sert de langue de communication lors des échanges commerciaux, et dont l'utilisation massive se fait au détriment de la pluralité des langues, et donc de cette « traduction » qui devrait pourtant être vue, en suivant de nouveau Eco (1993), comme la langue de l'Europe.

L'objet de ce point sur des questions anciennes autour de la traduction était de fournir un arrièreplan à notre réflexion sur les enjeux liés à cette pratique, et de mieux comprendre, grâce à cet éclairage historique, que la sacralisation (Cassin, 2014b) des traductions, liée à celle des langues, ne doit pas être une fin, et qu'une traduction est toujours en devenir. Ainsi, à la lumière de ce cadrage, nous allons à présent nous centrer sur les pratiques des traducteurs, et en particulier sur les manières, lorsqu'on traduit, de prendre en compte les spécificités des contextes, des personnes, des formes de vie (au sens de Wittgenstein ${ }^{3}$ ) dans lesquelles sont inscrites les systèmes d'énoncés.

\section{LES PRATIQUES DES TRADUCTEURS ET LA PRISE EN COMPTE DES CONTEXTES}

\section{Qu'est-ce qu'un traducteur?}

Le traducteur est une personne dont la pratique consiste à faire passer un énoncé ${ }^{4}$ dans une langue autre (sa langue maternelle, la plupart du temps) que celle dans laquelle il a été énoncé, afin que les personnes ne parlant pas cette langue soient en mesure d'accéder au sens de cet énoncé.

Il permet aux personnes non locutrices d'une certaine langue de résoudre un problème, ce problème étant leur rapport à un énoncé dont ils 
ne maîtrisent pas le « code » langagier. Résoudre ce problème, c'est donner à comprendre cet énoncé. Pour donner à comprendre cet énoncé, il faut donc maîtriser des connaissances langagières, mais pas seulement.

Afin d'être en mesure de répondre à cette question, il nous a semblé incontournable de faire appel à un praticien expert, un "savant» (Chevallard, 2007; Sensevy, 2011) de la traduction. En novembre 2014, nous avons donc conduit un entretien d'une vingtaine de minutes avec une traductrice littéraire chevronnée, spécialisée dans le domaine de la science-fiction, et ayant également été interprète pendant plusieurs années, en particulier des chefs d'État. La transcription de l'entretien, qui a été enregistré, figure en annexe. Au cours de cet entretien, nous avons questionné la traductrice sur ses pratiques de travail, sur les difficultés qu'elle était susceptible de rencontrer, et sur les stratégies lui permettant de traduire de la manière lui apparaissant la plus efficace possible. L'objectif d'un tel entretien était d'éclairer et de nourrir notre réflexion sur la pratique de la traduction avec les propos d'une " connaisseuse pratique » (ibid.), forcément tout autant à même que nous d'en appréhender les enjeux récurrents. Tout en gardant à l'esprit qu'un entretien avec une seule personne ne peut en soi garantir de nous faire connaître en profondeur la pratique de la traduction, nous inscrivons ce travail avec la traductrice dans une orientation de recherche plus générale, focalisée sur les pratiques des traducteurs, et dont cet article constitue un point de départ.

Ainsi, selon elle, être traducteur, " ce n'est pas une science, c'est un art». Le traducteur cherche à «trouver la voix de l'auteur» (ibid.) et à décoder ses intentions à partir d'indices contextuels. Cette voix de l'auteur, "c'est son style, la façon dont il s'exprime... il faut s'imprégner de son style pour pouvoir le restituer, en passant par ma voix à moi [celle de la traductrice], donc j'imprime aussi mon style » (ibid.). Cette voix dont parle la traductrice, ce sont des mots porteurs de sens qui, par leur récurrence ou leur positionnement dans le discours d'une personne, lui confèrent un style qui lui est propre, des manières caractéristiques.

C'est à ce style spécifique, au sens de « la façon d'exprimer un sujet » (Michel, 2004), que le traducteur doit accéder. Cet accès au style de l'énonciateur lui permet de saisir une certaine logique de style inhérente au discours qu'il a à traduire. Et c'est ensuite à la lumière d'un tel arrière-plan qu'il fait des choix, dans la langue d'arrivée, parmi des mots qui permettront d'établir une « logique de style » similaire au sein du discours traduit. Ainsi, en opérant tel ou tel choix, le traducteur « dit comme », ou «presque comme» (Eco, 2006) l'auteur.

\section{La temporalité}

Lorsqu'on traduit, on donne des formes à des systèmes d'énoncés, mais des formes qui ne sont jamais véritablement abouties, car stabiliser une traduction n'est pas possible. En traduction, la question de la temporalité, avec d'autres (culture, espace...), est essentielle. Par temporalité nous entendons l'inscription dans le temps de l'énoncé initial, de l'énoncé traduit, ainsi que l'évolution de ces énoncés dans le temps.

Par exemple, pour traduire un texte écrit il y a plusieurs siècles, il faut prendre en compte ce que revêtent les «institutions du sens » (Descombes, 1996) de l'époque. Ce qu'entend Descombes par cette expression, c'est que ce sont des "manières de faire et de penser », des « usages établis » (ibid.) qui sont, pourrait-on dire, les véritables « auteurs du sens des phrases ». Ce sont les usages établis au moment auquel les énoncés ont été produits qu'il faut prendre en compte en traduisant. Les équivalences qu'on instaure doivent tout à des conventions temporaires, écrit Rastier (2005). Ces usages établis font aussi bien entendu référence à d'autres formes de réseaux d'institutions du sens. Par exemple, pour traduire un prénom qui connote une certaine classe sociale, un certain mode de vie, les traducteurs doivent faire appel à des usages du langage qui font sens dans certains réseaux.

Ces usages établis peuvent déterminer des manières de faire et de penser très ponctuelles, et qui ne font pas forcément référence à des époques éloignées. L'établissement d'usages et d'institutions du sens est bien déterminé par des époques, des lieux, des sociétés, etc., mais aussi et surtout par les individus eux-mêmes. Ainsi que l'explique la traductrice interviewée, lors de son travail, elle recherche la « voix de l'auteur... son style, la façon dont il s'exprime ». Il s'agit bien d'institutions du sens propres à une personne et à son œuvre, même si cette "voix » s'inscrit bien entendu dans un contexte plus vaste. Ce sont alors cette voix et ce style qui, une fois 
décryptés par le traducteur au cours de son enquête, lui permettent de "voir comme» (Wittgenstein, 2004) l'auteur pour « dire presque comme » l'auteur. Sa pratique est, dans cette perspective, proche de celle de l'écrivain (De Launay, 2014).

L'auteur s'inscrit donc à la fois dans une certaine temporalité, dans une certaine culture, dans un certain langage, au sein desquels il établit ses propres manières de faire et de penser, et, pourrait-on dire, des institutions du sens ou, du moins, de sens institués, propres à l'ensemble de ses œuvres. La traductrice interviewée insiste d'ailleurs sur l'importance de la prise en compte du langage propre à l'auteur lorsqu'elle travaille. À la question « quelles sont vos stratégies pour traduire le plus efficacement possible? », elle répond qu'il lui faut « lire d'abord le livre [et] établir un lexique qui concerne les personnages et les mots inventés, les réalités, les univers inventés ». En prenant l'exemple du travail d'un médecin amateur d'art sur la peinture (Giovanni Morelli), Ginzburg (1989) parle de l'attention portée aux « petits détails matériels » au sein d'un « système de signes culturellement conditionnés [...] que les hommes, lorsqu'ils parlent ou écrivent, introduisent dans leur discours » (p. 171), et que Morelli qualifie de « traits purement individuels» (ibid., p. 146). La forme de la recherche, par la traductrice, des univers propres aux auteurs qu'elle traduit s'inscrit tout à fait dans cette perspective d'attention aux petits détails décrite par Ginzburg. Un traducteur, c'est donc quelqu'un qui mène une enquête.

Toujours à propos du langage de l'auteur, la traductrice interviewée parle également de l'importance de " connaître l'intrigue et situer les personnages et [de] connaître la fin », afin de " cerner sa voix ». Ici encore, la prise de connaissance de la patte de l'auteur, de son ouvre, est une étape clé dans le travail de traduction, qui consiste à décrypter certains jeux de langage (Wittgenstein, 2004) construits dans certains univers particuliers. On pourrait dire que le traducteur agit, à certains moments de son travail, à la manière d'un critique littéraire.

\section{Les variations individuelles, culturelles, sociales}

Maîtriser les manières de faire et de penser propres à l'auteur de l'énoncé à traduire, en lien avec les institutions qui donnent sens à son langage, est une stratégie indispensable lors de la traduction. Ces « institutions individuelles », pourrait-on dire, sont multi-factorielles: elles sont issues des expériences personnelles, culturelles, religieuses, sociales, etc., de chacun. Ce sont ces spécificités que le traducteur doit tenter de retracer afin de mieux saisir, et donc de donner à comprendre, le sens d'un système d'énoncés.

La traductrice interviewée donne l'exemple d'un auteur de science-fiction dont elle traduit régulièrement les ouvrages (William Gibson), et qui est à l'origine de l'utilisation de l'expression « espace inter-sidéral » pour décrire des sortes de mondes parallèles dans les galaxies. Elle explique que, pour donner naissance à ce terme en français et pour que cette expression produise le même effet sur le lectorat français que l'expression anglaise produit sur le lectorat anglophone, le traducteur de l'époque avait dû faire l'expérience du cheminement de l'auteur, chercher les sources de son inspiration: «Pour des termes comme l'espace sidéral ou intersidéral, il [l'auteur] s'inspire de quelque chose au départ, donc on essaie de retrouver son cheminement pour arriver à quelque chose d'équivalent en français... je sais de quoi ça a été inspiré, d'où ça vient, d'où vient l'inspiration, donc là je retourne à la source ».

Ce retour à la source montre bien un travail d'enquête, de recherche d'indices, qui vont permettre de décoder des jeux de langage particuliers. C'est également, dans un sens, un travail généalogique, qui est aussi celui qui doit être mené dans les sciences de la culture lorsqu'on travaille sur un concept ou une notion donnée.

Il s'agit aussi, pour le traducteur, de produire un certain effet, au sens d'Iser (1976), c'est-à-dire une expérience à faire vivre au lecteur. Comme le résume la traductrice:

« [Je dois] chercher à produire le même effet qu'en anglais. Qu'on peut arriver à comprendre. Là je suis sur un projet entre la science-fiction et la fantasy, je dois créer des mondes qui ont une origine, ça ne vient pas de nulle part, c'est à moi de recréer des termes qui auront le même effet en français qu'en anglais. »

Traduire, c'est tenter de produire une forme d'équivalence qui produirait le même effet dans une langue autre que celle dans laquelle l'énoncé a été initialement produit. Pour cela, la traductrice donne clairement à voir l'importance du jeu de langage, 
lorsqu'elle enquête sur les mondes créés par un auteur et lorsqu'elle crée à son tour des mondes qui sont " presque comme » ceux de l'auteur.

Lorsqu'on vise un certain effet en traduisant, on se situe dans une perspective fonctionnelle de la traduction, et non dans une démarche purement analogique. L'énoncé-source et l'énoncé-cible ont le même sens quand ils fonctionnent dans la même situation (Ladmiral, 2010). C'est dans cette perspective fonctionnelle que le traducteur opère des choix, ainsi que dans la supposition que le public-cible a une certaine familiarité avec l'auteur traduit (ibid.).

À présent, nous allons poursuivre le développement de nos arguments à l'aide de trois exemples, emblématiques de «l'intraduisible».

\section{ÉTUDE DE TROIS EXEMPLES « INTRADUISIBLES » : TRIEB, AVERROÈS, ET 磨课}

\section{Un premier exemple: Trieb}

Prenons l'exemple de la traduction du mot allemand Trieb, dont la traduction actuelle dominante est « pulsion». L'histoire de la traduction de ce mot fait l'objet d'un article dans le Vocabulaire européen des philosophies (Cassin, 2004), et ce qui suit reprend largement ce qui est dit dans cet article.

En langue française, le mot « pulsion » (un terme freudien, comme on le sait) s'articule autour d'une composante biologique (instinct), d'une composante physique (impulsion mécanique) et d'une « synthèse " naturaliste-romantique (force vitale). Depuis une centaine d'années, trois traductions successives de Trieb ont été proposées en français: jusqu'aux années trente, le mot « instinct » domine, auquel sera par la suite préféré le mot "pulsion », mais sans que cela implique clairement encore un rejet du lien avec la biologie; enfin, la polysémie du terme est acceptée par les traducteurs français à partir des années soixante. Ce détournement progressif correspond à une orientation stratégique, quasi-politique, ayant pour but de légitimer le projet intellectuel de la psychanalyse dans un entre-deux entre les univers de la biologie et de la psychologie. Le choix du sens donné à " pulsion » dans la dernière phase est parfaitement adapté à ce projet.

Ainsi, traduire Trieb, ce n'est pas traduire seulement un mot, mais aussi le « style de pensée » (Fleck, 1934 / 2005) qui l'accompagne.
L'exemple de Trieb donne bien à voir que les effets à produire sont inhérents au processus de traduction, aux choix opérés. Ces buts poursuivis ne sont pas insignifiants, puisqu'ils modifient les formes de certaines traductions, et par la suite les pratiques en lien avec les discours comportant ces traductions ${ }^{5}$. La pratique des psychanalystes n'est effectivement pas la même lorsqu'ils traitent des patients en voyant leurs pathologies comme des pulsions ou bien comme des instincts, car ils font alors des choix dans leur recours (ou non) à la biologie pour soigner les personnes. Dans le domaine judiciaire, l'instinct peut être qualifié de « sanguinaire » (article 211 du Code Pénal allemand), tandis que la pulsion est « sexuelle» ou « cupide» (ibid.), ce qui montre bien que le traitement des cas sera différent selon qu'on agit d'après ses pulsions ou ses instincts. C'est tout un « voir comme » (Wittgenstein, 2004) qui accompagne l'utilisation d'une traduction ou l'autre de ce mot.

L'histoire de la traduction de Trieb donne également à voir comment le choix d'une traduction au lieu d'une autre peut servir à légitimer le rapprochement ou l'éloignement d'un certain style de pensée, lié à une institution telle que la biologie ou la psychologie. Cela donne aussi à mesurer l'importance, lorsqu'on traduit, de la prise en compte du contexte historique, social, culturel, scientifique, etc., auquel il faut être vigilant afin de trouver la meilleure forme d'équivalence possible entre deux mots.

\section{Un deuxième exemple: Averroès et la traduction au cœur de la culture}

La traduction est un exemple de pratique particulièrement parlant du caractère indissociable de la langue et de la culture. Les formes que prend le langage sont des formes culturelles, et ces formes culturelles n'existent pas sans langage (au sens large). La traduction de la parabole du figuier dans la Bible ${ }^{6}$ en constitue un exemple frappant, car les discours bibliques nous sont parvenus à travers un certain nombre de traductions issues de précédentes traductions.

La traduction exemplifie bien cette détermination de notre pensée par les mots que nous employons afin d'exprimer un certain sens, en particulier lorsque nous nous trouvons face à des formes culturelles inconnues qu'il faut mettre en mots. Bazin (2008) dit par exemple que « comprendre une action c'est 
bien plutôt l'avoir décrite d'une manière telle qu'elle nous apparaisse comme l'une des manières possibles de faire selon d'autres règles ou dans d'autres conditions ce que nous-mêmes nous faisons » (p. 23). En cela, la pratique de la traduction s'inscrit tout à fait dans une perspective anthropologique.

Au cours de l'interview de Marc de Launay dans l'émission « Les Nouveaux chemins de la connaissance» (17 mars 2014), Adèle Van Reeth cite l'exemple d'Averroès. Eco (2006) fait également référence à l'histoire d'Averroès pour souligner sa " méprise culturelle », qui sera à l'origine de nombre de "méprises linguistiques » dans son travail sur Aristote, dont il commente les œuvres à partir de traductions. Dans « La quête d'Averroès » (1947), Borges décrit comment Averroès (un philosophe de langue arabe du XII ${ }^{e}$ siècle) n'est pas parvenu à traduire les termes « comédie » et « tragédie » de «La Poétique » d'Aristote. Averroès s'est trouvé en difficulté parce que la comédie et la tragédie, en tant que formes théâtrales spécifiques, n'existaient pas en tant que telles au XII ${ }^{\mathrm{e}}$ siècle dans la civilisation arabe ou, du moins, revêtaient des formes différentes de ce que comédie et tragédie décrivaient chez Aristote. En tant que formes culturelles spécifiques, la comédie et la tragédie sont caractérisées par des codes précis. Ce sont les codes qui régissent ces pratiques qui posent problème à Averroès, parce que ce qu'on peut entendre par «comédie " au sens large existait à l'époque dans les pays arabes, mais selon des codes et des formes bien différents de ceux qui caractérisaient les pratiques théâtrales décrites par Aristote.

Cet exemple donne également à voir l'articulation étroite des traductions avec le contexte social, culturel, géographique, et surtout temporel. En effet, à la manière de Trieb qui se traduit différemment au fil du XXe siècle en fonction des styles de pensées associées, la comédie et la tragédie, au fil des contacts entre l'Orient et l'Occident, seront davantage connues dans les pays arabes sous leurs formes décrites dans «La Poétique ». Pratiquer la comédie ou la tragédie c'est « une manière particulière d'être humain » (Bazin, 2008), mais selon un comportement social propre, lié à la "variabilité culturelle » (ibid.) que la description, et donc la connaissance de l'autre, peut amener à comprendre.

\section{L'intraduisible}

La question de "l'intraduisibilité », si l'on peut dire, est récurrente quand on s'intéresse à la pratique de la traduction. C'est d'ailleurs à travers l'étude de cette question qu'on en saisit la plupart des enjeux. Ce n'est pas un hasard si la plupart des exemples choisis, dans notre texte, comme dans la plupart des écrits sur le sujet, portent sur des «problèmes » de traduction. En effet, c'est lors de la résolution de ces problèmes que sont données à voir les stratégies et les arrière-plans convoqués, comme les références à l'histoire des mots ou les passages obligés par la production de descriptions des formes culturelles.

Pour traduire un terme problématique, il faut mettre au jour le jeu de langage auquel il appartient, la forme de vie (au sens de Wittgenstein) dans laquelle il s'inscrit. Ces jeux de langage peuvent être vus comme des réseaux (Cassin, 2014a) au sein des langues, mais aussi entre les langues. Pour trouver une forme d'équivalence entre deux termes, entre deux systèmes d'énoncés, il faut faire appel à la pratique culturelle qui fait fonctionner ces mots et trouver, au sein d'une autre pratique culturelle, des mots qui permettent de retranscrire une réalité plus ou moins équivalente. L'intraduisible n'existe pas en soi; tout est traduisible, bien qu'il existe des idées, des réalités, difficilement transposables d'une langue à l'autre. Dans le même sens, Bazin (2008) réfute l'idée d'altérité radicale, intraduisible, au profit de différences qu'il est toujours possible de « traduire » ou, du moins, de transposer à une autre culture, sous un certain angle. Il prend pour cela l'exemple de sacrifices africains (chez les Bambaras), que nous pourrions voir "presque comme» les rites d'une messe catholique. En effet, pour comprendre ces rites, nous avons en arrière-plan une idée de ce qu'est une cérémonie rituelle; "l'homme est un animal cérémoniel », dit d'ailleurs Bazin (ibid., p. 19) en référence à Wittgenstein. Cassin (2007), en décrivant la résolution de l'intraduisible dans une perspective d'enquête anthropologique, va dans le même sens:

« L'intraduisible ce n'est pas ce qu'on ne traduit pas. C'est ce qu'on ne cesse pas de traduire. Il ne faut pas sacraliser des intraduisibles qu'on devrait à tout prix maintenir en l'état [... ] Traduire c'est revenir sur l'épaisseur de l'histoire d'une langue: des emprunts, des héritages, des traces, des traumatismes...» (p. 31-42.)

« Il ne faut pas sacraliser des intraduisibles qu'on devrait à tout prix maintenir en l'état », car traduire 
ça peut être aussi produire une description, en passant par d'autres modalités que les signes verbaux. La traduction se situe donc à la croisée de formes de descriptions minces et épaisses (Ryle, 1971; Geertz, 1977 ; Descombes, 1998), selon qu'elle convoque ou non un certain style de pensée, et que le traducteur décide de décrire en une phrase ce qui dans une autre langue tient en un mot. C'est bien ce que dit la traductrice interviewée à ce sujet: «J'ai une expression dans le dernier livre que j'ai traduit, j'ai été obligée de faire toute une phrase pour l'expliquer pour rendre une notion, pour rendre l'expression ${ }^{7}$. ». Ainsi, en traduisant ce qui semble intraduisible, le traducteur contribue à réduire l'altérité, à transformer une étrangeté apparente $e^{8}$ en différence connue (Bazin, 2008), et ainsi à désacraliser ces éléments de langues dits « intraduisibles ». Cassin (2014b) insiste à juste titre sur la paganisation des langues et des traductions, à l'inverse d'une sacralisation telle que celle mise en place par les Grecs dans l'Antiquité autour du logos, ou encore de celle portée par Rivarol concernant la langue française. Cassin parle de la nécessité de ne pas voir les langues comme quelque chose de sacré et d'intouchable, afin de ne pas se laisser emporter par « le vertige de l'indicibilité essentielle de l'autre » (Bazin, 2008). En faisant cela, elle nous invite à considérer prioritairement la traduction comme une réflexion sur nos pratiques langagières et culturelles, qu'il convient de toujours questionner. Cette position est à rapprocher du travail de Quine sur l'indétermination de la traduction, ainsi que celui de Kuhn sur les paradigmes (2008), qui sont, tout comme la traduction, des formes de pensées, de significations ou de réalités indéterminées (Laugier, 2004).

\section{Un troisième exemple: 磨课}

En référence à des données filmées par Luc Trouche (2015) dans le cadre d'un projet de formation à la recherche entre les quatre ENS françaises et l'East Normal University de Shanghai (PROSFER ${ }^{9}$ ), nous allons prendre pour exemple la traduction du mot chinois 磨课 ( «mo-ke»). Lors d'un travail avec de jeunes chercheurs chinois en didactique des mathématiques, Luc Trouche (LT dans la transcription) leur a demandé s'il existait un terme lié à l'enseignement qu'ils trouvaient difficile à traduire en anglais. L'échange qui a suivi a été filmé, et nous en avons produit une transcription, dont l'extrait ci-dessous relate un échange avec une étudiante que nous avons appelée Yiran:

Tableau 1. Transcription de l'échange sur 磨课

\begin{tabular}{|c|c|c|}
\hline 10 & LT & $\begin{array}{l}\text { Oh oh oh! And Yiran how could you explain, for you, the content of this, the meaning of this word, of } \\
\text { this Chinese expression? }\end{array}$ \\
\hline 11 & Yiran & $\begin{array}{l}\text { For me it's a lesson preparation, with a circle period, just like a circle period } \\
\text { (dessine une hélice) and we have a beginning point but we have no ending point } \\
\text { You can improve this lesson again and again but there is no one who can never say "ok this lesson is } \\
\text { perfect" so we consider this (montre l'hélice) a circle period }\end{array}$ \\
\hline 12 & LT & OK, a sort of non-ended process \\
\hline 13 & Yiran & Yes, and it all depends on the teacher \\
\hline 14 & LT & And is it linked to a sort of collective work? I mean... \\
\hline 15 & Yiran & $\begin{array}{l}\text { Yes a collective work because when the teacher is giving this kind of lesson, maybe other members of } \\
\text { teachers' research group [?] They need to give the teacher some reflective ideas, or comments, or sugges- } \\
\text { tions }\end{array}$ \\
\hline
\end{tabular}


Suite aux échanges avec les étudiants, Trouche (2015) donne la traduction suivante de 磨课 (« mo-ke») :

« le processus de préparer une leçon sur le temps long, avec la volonté de la faire de mieux en mieux, avec la conviction que c'est un processus sans fin, et que c'est une responsabilité essentielle de l'enseignant, qui doit se nourrir des interactions avec ses élèves et ses collègues » (p. 1).

Nous avons également demandé à des personnes parlant chinois comment elles traduisaient ce mot. Une Française, enseignante de chinois depuis un certain nombre d'années, dont ce n'est pas la langue maternelle et qui vit en France, nous a indiqué ne pas comprendre ce mot et le traduire, à défaut par « étudiant non inscrit en université, qui suit des cours à distance ». Devant la difficulté à traduire ce mot, elle s'est en fait servie de la transcription phonétique du mot («mo-ke», prononcé « mooc»), à partir de laquelle elle a déduit qu'il s'agissait d'une forme de suivi de cours à distance, similaire à un MOOC $^{10}$.

Nous avons ensuite posé la question à une personne chinoise, vivant à Shanghai, et ne travaillant pas dans le domaine de l'éducation. Elle nous a donné la traduction suivante: 磨课 is used in education, aimed at improving the quality and effectiveness of teaching, with group works of teachers working on hot issues in teaching, repeatedly with in-depth study, research and practice. Sa conception de 磨课 est très proche de ce qui est décrit dans la transcription de l'échange filmé par Luc Trouche. Nous avons également demandé à cette même personne de nous indiquer ce à quoi renvoyait chacun des idéogrammes. Voici sa réponse: 磨 signifie "polir une pierre, donner une forme », et 课 est le nom qui désigne une classe ou une leçon. Pour donner à comprendre 磨, elle a joint à son explication l'image suivante:

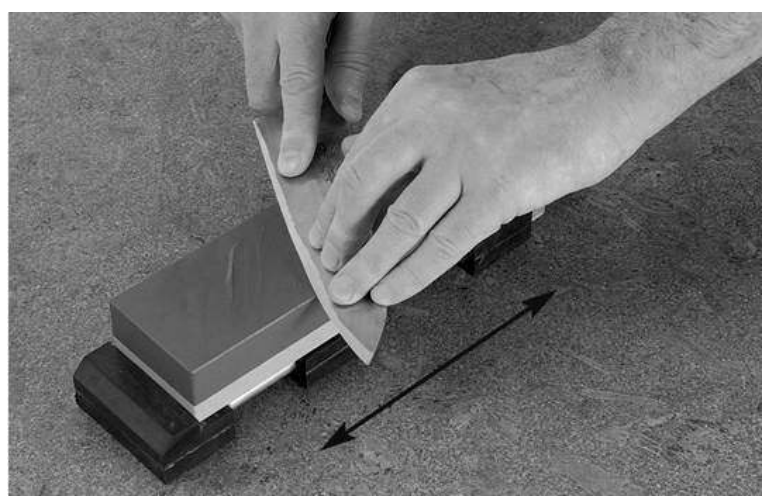

FIG 1. - Polir une pierre.

La difficulté à traduire ce mot, par ailleurs très emblématique de ce qu'est le travail d'un professeur d'un pays à l'autre, montre bien qu'il n'existe pas de mots intraduisibles et que c'est dans ces intraduisibles qu'on n'arrête pas de traduire qu'on trouve des descriptions épaisses (Ryle, 1971; Geertz, 1977; Descombes, 1998) de nos différences. Produire des descriptions anthropologiques, nous dit Bazin (2008), c'est " apprendre tout un monde ": le symbole de la pierre à polir montre bien que, pour saisir le cœur de l'expression 磨课, il faut passer par la découverte d'un monde qui ne se décrit pas forcément qu'avec des mots, mais aussi à l'aide d'images. Il s'agit ici d'une forme de traduction inter-sémiotique décrite par Jakobson (1959).

Ainsi, traduire, c'est produire des descriptions et, ce faisant, prendre en compte des pratiques langagières, culturelles: c'est donc une véritable démarche anthropologique. Le fait que l'enseignante française de chinois ne puisse pas traduire ce mot, alors que la personne chinoise y parvient, met également en lumière l'importance du "monde » qui entoure les mots. Peut-être le terme 磨课 est-il récemment employé dans la société chinoise, parce que des recherches sont en cours depuis peu sur le travail des professeurs, ce qui expliquerait la difficulté de l'enseignante française à traduire ce mot qu'elle n'aurait pas eu l'occasion de «fréquenter » lors de sa formation initiale. D'ailleurs, Luc Trouche demande, à la fin de l'échange avec l'étudiante chinoise, si ce terme est compris en Chine, en dehors du monde de la recherche: 
Tableau 2. Suite de la transcription de l'échange sur 磨课

\begin{tabular}{|l|l|l|}
\hline 29 & LT & $\begin{array}{l}\text { In China. And is it a common word } \\
\text { for the Chinese society? I mean if } \\
\text { you show this word to a man in the } \\
\text { street, he will understand what it } \\
\text { means? }\end{array}$ \\
\hline 30 & Yiran & Yes, as I know, yes. \\
\hline
\end{tabular}

Cet exemple donne aussi à voir que la traduction d'un terme problématique ne se résout pas uniquement à l'aide de signes verbaux: le dessin de l'hélice pour symboliser le cycle de travail permanent du professeur qui prépare et reprend ses séances est un appui fort à la description de cette pratique. Traduire, c'est aussi, au sens large, produire une description, qu'elle soit à l'aide de signes verbaux ou non verbaux, qui permette d'engager une réflexion sur les langues, sur les pratiques culturelles, et d'ouvrir le dialogue avec l'autre.

Cet exemple montre combien la traduction qui, vue comme une négociation permanente entre les langues, peut nous permettre de repenser des allant-de-soi, voire de renouveler nos pratiques. Les langues, rappelle Castelloti (2010), c'est ce qui permet de se transformer au contact d'autrui.

À la lumière des arguments développés, et de la conception de la traduction que nous avons esquissée, nous allons maintenant nous focaliser sur les enjeux qu'une telle vision de la traduction pourrait impliquer en classe de langue vivante à l'école.

\section{QUELLE CONTRIBUTION CETTE RÉFLEXION APPORTE-T-ELLE À LA QUESTION DE LA TRADUCTION EN DIDACTIQUE DES LANGUES?}

À l'appui de notre réflexion générale sur la traduction, l'objectif de cette partie sur la didactique des langues est de réfléchir aux enjeux liés à cette pratique en classe de langue vivante, à la lumière de l'arrière-plan conceptuel établi au fil de notre article.

\section{La place de la traduction à l'école}

$\mathrm{Au}$ sein des institutions scolaires, la traduction n'est pas une pratique très répandue. En classe de langues vivantes, elle n'est pas véritablement prise en considération en tant que technique de travail sur la langue. En fait, c'est une pratique qui existe surtout sous la forme des exercices classiques de thème et de version, qui sont des exercices écrits visant essentiellement à évaluer des connaissances d'ordre syntaxique et lexical, à contrôler un certain niveau de langue, et à montrer au professeur ce qu'on sait en langue vivante (Ladmiral, 2010). Nous pensons d'ailleurs qu'il existe un risque réel à ce que l'évaluation « pervertisse » la pratique de la traduction à l'école.

La pratique de traduction ne fait donc pas l'objet d'un travail en soi, c'est-à-dire que les techniques, les stratégies (comme les références à l'histoire des mots, aux formes culturelles) qui lui sont liées, ne sont pas véritablement travaillées. La traduction dans les pratiques scolaires n'est ainsi pas une fin en soi mais plutôt un moyen, notamment un moyen d'évaluer.

Au sein de cette visée évaluative, c'est d'ailleurs la perspective du bilinguisme qui est davantage mise en avant, que celle de la pratique traductrice, bien que les deux soient liées. L'idée derrière cette perspective est la suivante: une personne bilingue est forcément performante en traduction. De Pietro (2013) dénonce ainsi le fait que « trop souvent, malheureusement, la traduction [soit] appréhendée comme un exercice qui serait fondé sur la maîtrise la plus approfondie possible des langues concernées » (p. 234). Cette position peut aussi laisser entendre que les élèves peu ou moyennement avancés dans leur apprentissage d'une langue ne peuvent pas pratiquer la traduction, ou que cette pratique les placera en situation d'échec. Ce qui est effectivement le cas si la pratique dont il est question porte sur des exercices de thème et de version, visant à évaluer exclusivement un certain niveau de langue.

Par ailleurs, afin de compléter notre point de vue, une enquête sur les pratiques en classe de langue allant dans le sens de la conception de la traduction que nous proposons, pourrait constituer un axe d'étayage futur tout à fait fructueux à développer.

\section{Les Approches Plurielles de l'enseignement des langues}

Afin de dépasser cette visée exclusivement évaluatrice de la traduction, De Pietro (ibid.) propose de " développer une didactique de la traduction " (ibid.), qui serait fondée sur les descripteurs du Cadre de Référence pour les Approches Plurielles (CARAP), développé par Candelier (2010), à la suite des travaux portant sur l'Éveil aux Langues (Candelier, 
2003). Aden et Leclaire (2013) présentent ainsi les idées clés de l'Éveil aux Langues:

"L'éveil aux langues permet de percevoir intellectuellement et d'accepter que les langues traduisent les manières différentes dont les humains perçoivent le réel dans l'action sociale [...] Ce ne sont pas les connaissances sur les langues qui priment mais les liens qu'il faut imaginer, les chemins qu'il faut parcourir ensemble, pour qu'émerge un sens partagé [...] Même si nos cultures ont inventé des mots, des rythmes et des structures différents pour dire nos sensations, nos émotions, nos affects, et même si nos cultures ont développé des stratégies collectives différentes, traduites le plus souvent en règles intériorisées, nous négocions un sens partagé à partir de notre expérience sensorielle du monde, y compris dans la conceptualisation mathématique. » (Aden \& Leclaire, 2013, p. 145.)

Faire en sorte que les élèves se questionnent sur le fonctionnement des langues est un élément central de cette approche. Les faire enquêter sur des mots, des expressions, dits "intraduisibles", et pour lesquels il est nécessaire « d'apprendre tout un monde " (Bazin, 2008), nous semble être une approche qui ferait appel à des réseaux de compétences très variés et, de ce fait, à la fois proche de ce qui fait la pratique des « savants » de la traduction, et permettant de développer des capacités métalinguistiques indispensables.

Les descripteurs dont parle De Pietro (2013) dans le CARAP sont les suivants:

« savoir que la traduction d'une langue vers une autre peut rarement se faire terme à terme, comme un changement d'étiquettes, mais s'inscrit nécessairement dans un découpage différent de la réalité (K6. 2. 2); savoir qu'à un mot du lexique d'une langue peuvent correspondre deux ou plusieurs mots dans une autre langue (K6. 2. 2); être capable d'accéder au moins partiellement au sens d'un énoncé dans une langue peu ou pas connue sur la base d'un repérage des mots et d'une analyse de la structure syntaxique/ morphosyntaxique (S1. 4. 3)» (p. 234).

Ces descripteurs ont été produits dans le cadre du développement des approches plurielles, également appelées approches plurilingues (Moore, 2006), en lien avec l'enseignement des langues, notamment à l'école primaire. Ils ont pour but de proposer des alternatives à l'enseignement isolé d'une langue, et d'impliquer, à travers des activités de réflexion sur les langues, une plus grande variété linguistique et culturelle dans cet enseignement (Troncy, 2013).

La référence, dans le descripteur S1. 4. 3, au repérage de certains mots, ainsi qu'à l'analyse de la structure d'un énoncé, propose une vision similaire à la conception de la traduction que nous avons développée au fil de l'article, qu'on pourrait voir comme la résolution d'une enquête sur le langage. La recherche d'indices syntaxiques, l'appui sur le repérage de certains mots, sont des manières d'appréhender la traduction en classe de langue qui s'inscrivent dans cette perspective.

Candelier, Kervran et Rémy-Thomas (2003), avaient également proposé, dans le cadre de l'Éveil aux Langues, de faire travailler les élèves d'école primaire sur la découverte de langues autres que celles préconisées par les programmes, dans le but de leur faire développer des aptitudes métalinguistiques (entre autres). Ainsi, ils proposaient, à travers l'étude de systèmes alphabétiques éloignés les uns des autres, un travail d'enquête sur les sons, sur les mots, sur les structures de phrases, avec un passage par l'émission d'hypothèses quant aux langues et aux locuteurs utilisant ces systèmes alphabétiques. Ce type de démarche est proche de la conception de la traduction que nous esquissons, et il remet en cause (Kervran, 2011) la conception de la traduction comme une équivalence terme à terme.

Par exemple, l'activité ci-dessous, issue d'un ensemble de documents proposés dans le cadre de l'Éveil aux Langues, place l'activité de traduction des élèves au sein d'une démarche d'enquête:

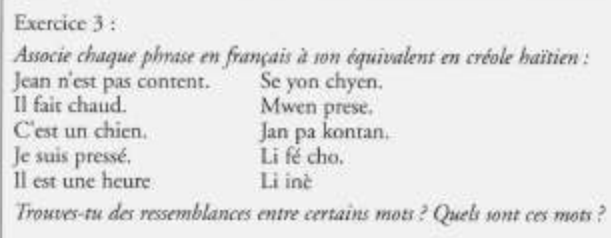

FIG. 2. - Correspondance français-créole (Candelier, Kervran E Rémy-Thomas, 2003).

Ainsi, faire enquêter les élèves sur des formes langagières et culturelles sur lesquelles il convient de toujours se réinterroger, parce que les traductions ne sont pas figées, c'est aussi introduire dans les 
pratiques des professeurs de langues une vision du traitement de la langue autre que celle associée au «perfectionnisme [qui] détermine encore largement les pratiques professorales »(Gruson, 2006, p. 614).

Actuellement, les programmes pour l'enseignement et l'apprentissage d'une langue étrangère à l'école primaire n'évoquent pas la traduction. Ce n'est pas réellement le cas au collège et au lycée non plus, bien qu'il soit question au collège de réflexion sur l'origine et le sens des mots importés d'une langue à l'autre. Peut-être est-il permis d'espérer que cette pratique soit un jour mieux intégrée en classe de langue, et dans une perspective renouvelée:

«En se fondant sur un nouveau rapport à la grammaire / traduction, il se pourrait bien que l'ancien libellé d'enseignement d'une langue étrangère à l'école devienne l'enseignement des langues aux enfants. » (Candelier, Kervran \& Rémy-Thomas, 2003, p. 65.)

\section{La traduction comme une enquête à faire mener par les élèves}

Les exemples proposés dans le cadre des Approches Plurielles présentent une vision de la traduction plus réflexive que la perspective dans laquelle cette activité se place actuellement à l'école. Ils impliquent un travail sur plusieurs langues et, en particulier, sur des langues qui ne sont pas enseignées à l'école.

Faire enquêter les élèves sur le langage, la culture, sur la résolution des « intraduisibles », peut en effet être un travail fructueux dans le cadre de l'apprentissage des langues vivantes, permettant de faire appel à la réflexion métalinguistique. C'est aussi une pratique qui pourrait trouver sa place, de manière plus légitime qu'actuellement, dans le cadre de l'intégration des élèves n'ayant pas le français comme langue maternelle, et dont la langue d'origine est généralement perçue institutionnellement comme un problème, et non un atout pour l'apprentissage (Moore, 2006).

Nous pensons également, pour notre part, qu'une conception de la traduction au sens d'enquête anthropologique telle que nous l'avons esquissée, représenterait une forme de travail sur la langue tout à fait profitable même dans le cadre de la découverte d'une seule langue, et pas uniquement dans le cadre d'approches plurilingues. En effet, la pratique de la traduction, au sens de mise en relation entre des formes de langue, de culture, des temporalités, etc., nous semble propice à développer chez les élèves des capacités de prises en compte plus générales des passages possibles entre les langues, entre les cultures quelles qu'elles soient. La remise en cause de la conception de la traduction terme à terme (Kervran, 2011) peut également être mise en place dans le cadre plus traditionnel de l'enseignement d'une seule langue. Car c'est en fait avant tout une autre conception du travail de traduction qu'il faudrait repenser pour l'école.

\section{EN CONCLUSION}

Traduire c'est, à travers la prise en compte des spécificités de contextes, de personnes, la résolution d'une enquête sur le langage, qui contribue, pour reprendre les propos précédemment cités de Bazin (2008), à transformer une étrangeté apparente $^{11}$ en différence connue. Traduire, c'est aussi, d'un certain point de vue, produire des descriptions anthropologiques:

« Le travail anthropologique - travail critique plus que jamais à l'ordre du jour - n'est pas de promouvoir l'altérité, mais de la réduire. Si étranges, voire parfois absurdes, que nous paraissent d'abord des actions humaines, il doit y avoir un point de vue d'où, une fois mieux connues, elles se révèlent seulement différentes des nôtres: c'est en quoi leur description est anthropologique.» (ibid., p. 22.)

Les propos de Bazin permettent de comprendre que la production de descriptions, en tant que pratique anthropologique, est organiquement tournée vers la compréhension d'autrui. Ainsi, pour compléter les propos de Steiner (1998) qui déclare que "comprendre, c'est traduire», nous pouvons ajouter qu'inversement, traduire, c'est aussi comprendre, et en particulier comprendre certaines formes de vie (au sens de Wittgenstein).

À la croisée des pratiques de l'écrivain et du critique littéraire, le traducteur mène une enquête qui aboutit à une situation ponctuellement déterminée - car elle permet de résoudre un problème mais dont la détermination ne doit pas être considérée comme intouchable. De la même manière que les paradigmes de Kuhn (2008) sont à voir, nous 
l'avons dit précédemment, comme des formes de pensées indéterminées (Laugier, 2004), la traduction ne doit pas être sacralisée et considérée comme un produit fini; ce qui est d'autant plus important que, si on pousse le parallèle avec Kuhn un peu plus loin, la pratique de la traduction permet, tout comme les paradigmes, d'appréhender une certaine forme de pratique, langagière, culturelle, etc. Lorsqu'on traduit, on projette finalement un certain « voir comme " (Wittgenstein, 2004) des systèmes d'énoncés à traduire. Par la suite, le traducteur donne aussi à voir, à celui qui lit ou écoute sa traduction, son propre « voir comme » des systèmes d'énoncés traduits. La paganisation des langues (Cassin, 2014b) et des traductions, c'est-à-dire le refus de les voir comme des œuvres finies et quasi-sacrées, est une perspective indispensable au renouvellement des formes de pensées. Mais alors, que répondre à la question «qu'est-ce que traduire »?

Traduire, c'est faire appel à un vaste répertoire de connaissances et de compétences langagières et culturelles (au sens très large) pour comprendre un discours, auquel on donne une autre forme tout en essayant d'en conserver le sens, mais en étant conscient que traduire est un travail perpétuellement inabouti. Notre vision, qui n'est que l'esquisse d'une certaine conception, donne donc à voir la traduction comme une pratique de réflexion sur les langues en présence.

Empruntons pour conclure ces paroles à Anne Coldefy-Faucard, traductrice de Nicolas Gogol, qui résume en ces quelques phrases en quoi consiste son travail:

«Bien sûr qu'elle vieillira. Le traducteur a beau faire attention, il est très influencé par son époque. Il y a toujours des choses qui se mettent à sonner faux. Même Pouilleuse-la-Ramène ${ }^{12}$ dont j'étais tellement contente, peut-être que dans 10 ans ce sera ridicule. Il faudrait refaire les traductions tous les vingt ans. Il faut les refaire parce qu'on met trop de soi dans une traduction, et de soi au moment où l'on traduit. Une traduction, c'est avant tout la lecture que fait un traducteur d'une œuvre. Si j'avais traduit Le Manteau à quinze ans, je ne l'aurais pas traduit de la même façon, j'en aurais fait une lecture complètement différente. On change, on évolue soi-même, on agrandit son lexique, sa compréhension des choses. On ne met plus les accents aux mêmes endroits... Et c'est souvent une toute petite nuance qui décide de tout. » (2007, p. 93.) 


\section{ANNEXE}

Transcription de l'entretien avec la traductrice

\begin{tabular}{|c|c|c|}
\hline 1 & Cherch. & Qu'est-ce que c'est traduire pour vous? \\
\hline 2 & Trad. & $\begin{array}{l}\text { Je me définis comme un passeur, c'est-à-dire la personne qui sert d'intermédiaire entre deux langues. } \\
\text { Je suis traductrice littéraire. Quand je traduis mon rôle c'est de privilégier la langue cible c'est-à-dire } \\
\text { de produire de produire un travail qui sera lisible en français, comme s'il avait écrit en français donc ça } \\
\text { demande énormément d'adaptation du texte de départ } \\
\text { Le but du jeu pour moi c'est de produire un texte en français, comme s'il avait été rédigé en français. }\end{array}$ \\
\hline 3 & Cherch. & Vous traduisez quelles langues? \\
\hline 4 & Trad. & $\begin{array}{l}\text { De l'anglais vers le français; dans le cadre de mes études en tant qu'interprète, j’interprète de l'espa- } \\
\text { gnol vers le français. Je travaille essentiellement de l'anglais vers le français. Moi j’ai deux langues } \\
\text { maternelles, je suis complètement bilingue, bi-culturelle, j'ai également deux nationalités, française et } \\
\text { canadienne. }\end{array}$ \\
\hline 5 & Cherch. & $\begin{array}{l}\text { Comment vous évaluez vos traductions? Qu'est-ce qui détermine une bonne traduction? Qu'est-ce qui } \\
\text { fait que vous êtes satisfaite d'une traduction et que vous la trouvez réussie? }\end{array}$ \\
\hline 6 & Trad. & $\begin{array}{l}\text { Je la fais relire par mon mari, quand lui comprend et n'est pas choqué. C'est très difficile pour le tra- } \\
\text { ducteur de juger lui-même; c'est presque mission impossible pour un traducteur de se relire et d'être } \\
\text { satisfait de son travail. C'est pas une science, c'est un art. }\end{array}$ \\
\hline 7 & Cherch. & $\begin{array}{l}\text { Est-ce que vous pensez que c'est possible qu'une traduction soit aboutie? Ou c'est toujours quelque } \\
\text { chose qui est à retravailler? }\end{array}$ \\
\hline 8 & Trad. & $\begin{array}{l}\text { Je pense qu'une traduction pourra toujours être retravaillée parce que ça dépend du point de vue. } \\
\text { Deux relecteurs différents trouveront toujours des choses différentes, et comme je passe derrière le } \\
\text { relecteur, je trouve aussi encore des choses. Je pense qu'il est pas possible de faire des choses défi- } \\
\text { nitives, on l'a vu avec des grands romans comme Moby Dick qui vient d'être retraduit parce que la } \\
\text { langue évolue, la langue change, la langue d'il y a } 50 \text { ans elle n'est pas très agréable à lire aujourd'hui. }\end{array}$ \\
\hline 9 & Cherch. & Quelle différence vous faites entre un traducteur littéraire et technique? \\
\hline 10 & Trad. & $\begin{array}{l}\text { Largent. En littéraire on doit trouver la voix de l'auteur et essayer de rendre la voix de l'auteur, s'il avait } \\
\text { écrit en français comment il aurait dit ça ou ça. En technique on colle beaucoup plus, on peut presque } \\
\text { faire une traduction informatique et avec des ajustements on y arrive. }\end{array}$ \\
\hline 11 & Cherch. & Vous utilisez des logiciels? \\
\hline 12 & Trad. & $\begin{array}{l}\text { Non je travaille en fantasy et SF et dans ce domaine les logiciels ne servent pas les nouveaux domaines } \\
\text { créés pas les auteurs; il faut créer des nouveaux mots. }\end{array}$ \\
\hline 13 & Cherch. & Vous créez des nouveaux mots? Vous faites comment? \\
\hline 14 & Trad. & $\begin{array}{l}\text { Il faut que ça corresponde à quelque chose; l'auteur a créé quelque chose qui vient de quelque part, } \\
\text { il a toujours des sources. J'ai travaillé avec William Gibson, qui est le pape d'un nouveau genre de } \\
\text { science-fiction, et il a créé des termes comme l'espace sidéral ou inter-sidéral, c'est lui qui a créé ces } \\
\text { termes-là en anglais, donc il s'inspire de quelque chose au départ, donc on essaie de retrouver son che- } \\
\text { minement pour arriver à quelque chose d'équivalent en français, c'est très amusant à faire mais parfois } \\
\text { on s'arrache les cheveux. }\end{array}$ \\
\hline 15 & Cherch. & Le but de cette re-création c'est d'arriver à produire un effet? Le même effet? \\
\hline 16 & Trad. & $\begin{array}{l}\text { Chercher à produire le même effet qu'en anglais. Qu'on peut arriver à comprendre. Là je suis sur un } \\
\text { projet entre la SF et la fantasy, je dois créer des mondes qui ont une origine, ça ne vient pas de nulle } \\
\text { part, c'est à moi de recréer des termes qui auront le même effet en français qu'en anglais. }\end{array}$ \\
\hline 17 & Cherch. & Du coup vous aussi vous menez une espèce de cheminement, une enquête en fait? \\
\hline 18 & Trad. & $\begin{array}{l}\text { Oui. Dans l'histoire de la SF, ce sur quoi je travaille, en fait, je sais de quoi ça a été inspiré, d'où ça } \\
\text { vient, d'où vient l'inspiration, donc là je retourne à la source, qui a une cinquantaine d'années donc } \\
\text { c'est assez facile de trouver de la documentation. }\end{array}$ \\
\hline 19 & Cherch. & Tout à l'heure vous avez parlé de la voix, donc vous cherchez à retrouver son intention. \\
\hline
\end{tabular}


Carole Le Hénaff

\begin{tabular}{|c|c|c|}
\hline 20 & Trad. & $\begin{array}{l}\text { Sa voix c'est son style, la façon dont il s'exprime, donc il faut bien cerner sa voix. Il faut d'abord lire } \\
\text { deux fois le livre pour connaître l'intrigue et situer les personnages et il faut connaître la fin pour ne } \\
\text { pas faire fausse route. Il faut connaître l'intrigue et s'imprégner du style de l'auteur pour restituer le } \\
\text { style de l'auteur, en passant par ma voix à moi, donc j'imprime mon style sur le livre. }\end{array}$ \\
\hline 21 & Cherch. & Quelles sont les ressources que vous utilisez au quotidien? \\
\hline 22 & Trad. & $\begin{array}{l}\text { Moi j'utilise énormément Google ou Wikipédia pour vérifier les occurrences des expressions, si elles } \\
\text { sont courantes. J'utilise Longman Online, un dictionnaire britannique et le Petit Robert pour vérifier } \\
\text { la traduction, les dictionnaires de synonymes. Quand je n'ai pas accès à Internet je suis mal. Je me fais } \\
\text { des glossaires, avec Excel par exemple. }\end{array}$ \\
\hline 23 & Cherch. & Quelles sont vos stratégies pour traduire le plus efficacement possible? \\
\hline 24 & Trad. & $\begin{array}{l}\text { Lire d'abord le livre. Établir un lexique qui concerne les personnages et les mots inventés, les réalités, } \\
\text { les univers inventés. Ensuite premier jet, ensuite relecture avec recul. Ensuite } 2 \text { ou } 3 \text { relectures défini- } \\
\text { tives. Je fais relire; beaucoup de traducteurs font relire leur famille pour ne pas trop coller au texte. }\end{array}$ \\
\hline 25 & Cherch. & $\begin{array}{l}\text { Comment faites-vous quand vous tombez sur un mot que vous trouvez extrêmement difficile à tra- } \\
\text { duire, qui est l'ordre de l'intraduisible? Quelle est votre stratégie? }\end{array}$ \\
\hline 26 & Trad. & $\begin{array}{l}\text { J'ai une expression dans le dernier livre que j'ai traduit, j'ai été obligée de faire toute une phrase pour } \\
\text { l'expliquer, pour rendre une notion, pour rendre l'expression. Des fois ce sont des néologismes. Le } \\
\text { contact avec l'auteur est possible, par exemple lorsqu'on parle de quelque chose qui n'existe pas en } \\
\text { France. Un auteur parlait d'un gamin qui volait des lecteurs de cassettes dans des voitures. Il s'agissait } \\
\text { de très grosses cassettes n'ayant jamais existé en France. J'ai contacté l'auteur et je lui ai demandé si } \\
\text { on pouvait parler de lecteur de cassettes normal. Il y a toujours possibilité de prendre contact avec } \\
\text { l'auteur. }\end{array}$ \\
\hline 27 & Cherch. & Est-ce que vous avez d'autres choses à ajouter qui vous paraissent essentielles sur votre activité? \\
\hline 28 & Trad. & $\begin{array}{l}\text { Je fais facilement des paraphrases, expliquer un terme plutôt que le traduire, quand je ne trouve pas. Je } \\
\text { trouve que ça se sent dans mes traductions parce que je suis interprète. }\end{array}$ \\
\hline 29 & Cherch. & Est-ce que vous pourriez décrire comment vous faites quand vous interprétez? \\
\hline 30 & Trad. & $\begin{array}{l}\text { Je ne sais pas. On se met en mode hyper-concentré, on a des mémoires à court terme extrêmement } \\
\text { développées mais quand on sort de cabine, on ne sait plus, parfois, de quoi les intervenants ont parlé, } \\
\text { parce que c'est la mémoire à court terme qui prend le dessus. Donc on se découvre une oreille pour } \\
\text { s'écouter soi-même travailler, et se critiquer. Donc il faut s'entendre soi-même. On entend d'une oreille, } \\
\text { j'entends l'intervenant avec mon oreille gauche et j'analyse le discours de l'intervenant. Les interprètes } \\
\text { n'ont pas un dictionnaire anglais-français dans la tête donc on passe par le concept. Un exemple si } \\
\text { j'entends le mot « chair », je n'ai pas un dictionnaire dans ma tête qui me dit « chair » = « chaise », } \\
\text { je vois l'image dans ma tête d'une chaise qui surgit immédiatement et je re-décris l'image que je vois } \\
\text { avec l'autre lexique dans lequel je travaille. J'ai deux lexiques en tête, qui me servent à décrire. Ça } \\
\text { vaut pour les concepts, les idées, et tout ce qui est abstrait aussi. Donc j'écoute le message de l'oreille } \\
\text { gauche, je traduis en concepts, en notions, en images et je le re-décris par la voix dans l'autre lexique } \\
\text { tout en écoutant et en analysant mon travail. Il faut s'entendre parler. Il faut je puisse me concentrer } \\
\text { sur deux choses en même temps, le message reçu et le message délivré, le message que moi je rends. } \\
\text { Il y a la concentration sur le message, l'analyse du message et le rendu. En même temps que je parle } \\
\text { je continue de me concentrer sur ce que j'entends. C'est un nombre infini de choses que mon cerveau } \\
\text { traite en même temps. }\end{array}$ \\
\hline
\end{tabular}

26 novembre 2014. Durée: environ 20 minutes. 


\section{NOTES}

1. C'est d'ailleurs une des compétences décrites dans les niveaux du Cadre Européen de Référence pour les Langues de 2001, qui hiérarchise le niveau d'un locuteur dans une langue seconde en fonction de critères, dont la capacité à être un médiateur, c'est-à-dire à produire à destination d'un tiers la reformulation d'un énoncé auquel ce tiers n'a pas d'accès direct.

2. Iser (1976), par exemple, insiste sur l'effet produit sur le lecteur d'un texte, au sens « d'expérience que le texte fait vivre au lecteur $»$ (Marzloff, 1999).

3. Voir Glock, 2003, p. 250-257.

4. Le terme «énoncé » est à prendre au sens de « système d'énoncés » dans notre texte.

5. Dans le même esprit, Descombes (1996) donne l'exemple des éléphants: "Grâce à ce mot [éléphant], l'éléphant entre dans les délibérations des hommes. Or ces discours ont conduit à des décisions concernant les éléphants [...] le destin des éléphants dépend beaucoup plus de leur histoire intentionnelle - celle qu'ils ont dans l'esprit et dans les paroles des hommes - que de leur histoire naturelle. » (p. 44-45.)

6. Parabole sur la stérilité d'un figuier, citée par Ladmiral (2010), dont il existe des espèces comestibles en Europe, mais vénéneuses en Amérique centrale.

7. Elle explique, suite à une demande de précision de notre part, qu'il s'agit de l'expression anglaise however twisted, qu'elle traduit par « votre conception tordue de l'honneur vous fait placer la mission avant tout. »

8. Nous sommes d'ailleurs déjà, en utilisant notre propre langue, dans un rapport d'étrangeté vis-à-vis de cette langue, car elle ne nous appartient pas et nous pré-existe (Cassin, 2014b). Ceci explique qu'on soit aussi dans un certain rapport d'étrangeté à notre soi, et que notre soi puisse devenir « milieu » (Sensevy, 2011), quand on fait de l'art ou du sport, mais aussi quand on parle une langue étrangère.

9. PROgram of Sino-FRench. Il s'agit d'un programme de formation à la recherche impliquant les quatre ENS françaises et l'ECNU (East Normal Universityà à Shangai.

10. Massive Open Online Courses (cours universitaires en ligne, gratuits, et connaissant un fort développement depuis peu).

11. Il arrive toutefois que cette étrangeté soit prise en compte par les traducteurs, notamment lorsqu'ils traduisent des écrits ayant un style littéraire très spécifique, comme le poème Le Bateau Ivre de Rimbaud, dont le rythme faussement désorganisé contribue à faire perdre ses repères au lecteur (Boisseau, 2010).

12. Nom donné par la traductrice au village nommé Vchivaïa Spes par Nicolas Gogol dans Les Âmes Mortes (1842)

\section{RÉFÉRENCES}

Aden, J., \& Leclaire, F. (2013). Éveil aux Langues et théories de la complexité: reconfigurations linguistiques et identitaires. Dans C. Troncy (dir.), Didactique du plurilinguisme. Approches plurielles des langues et des cultures. Autour de Michel Candelier (p. 141-148). Rennes, France: Presses universitaires de Rennes.

Bazin, J. (2008). Des clous dans la Joconde. L'anthropologie autrement. Toulouse, France: Anarchsis.

Boisseau, M. (2010). Beckett, Mahon, Carson et Le Bateau Ivre: Rythme, prosodie, interprétation. Études Irlandaises, 35, 135-148.

Borges, J. L. (1947). La quête d'Averroès. Dans J.-P. Bernès (dir.), L'Aleph, Euvres Complètes (p. 615-623). Paris, France: Gallimard.

Candelier, M. (dir.) (2003). Léveil aux langues à l'école primaire. Evlang: bilan d'une innovation européenne. Bruxelles, Belgique: De Boeck.

Candelier, M., Kervran, M., \& Rémy-Thomas, F. (2003). Une approche plurielle des langues à l'école primaire. Construire de nouvelles compétences préparatoires à la traduction. Le français aujourd'hui, (142), 55-67.

Candelier, M. (2010). Le Cadre de Référence pour les Approches Plurielles des Langues et des Cultures (CARAP) - Un outil pour la prise en compte de la diversité à l'école. Politiques et pratiques de l'enseignement et de la diversité socioculturelle, (4), 85-93.

Cassin, B. (2007). Le plaisir de traduire. Geste, Traduire, (4), 31-42.

Cassin, B. (dir.). (2004). Vocabulaire européen des philosophies. Paris, France: Éditions du Seuil.

Bernard, A., \& Naud, H. (2009). La cyberdépendance [Reportage]. Dans A. Naud (Réalisatrice), Découverte. Montréal, Québec: Société Radio-Canada.

Cassin, B. (2014a). La traduction: que faire des concepts intraduisibles?, France Culture, Les Nouveaux Chemins de la Connaissance, émission du 20 mars. Repéré à: http:// www.franceculture fr/emission-les-nouveaux-chemins-de-la-connaissance-la-traduction-44-que-fairedes-concepts-intraduisib

Cassin, B. (2014b, novembre). Compliquer l'universel. La traduction comme nouveau paradigme des sciences humaines. Conférence présentée à la journée d'étude du CNRS « Philosopher en Langues, 10 ans d'intraduisibles », Paris. France.

Castelloti, V. (2010). Enseignement du français et plurilinguisme au Japon: pour une didactique contextualisée. Revue japonaise de didactique du français, (5), 9-27.

Chevallard, Y. (1991). La Transposition didactique. Grenoble, France: La Pensée Sauvage.

Chevallard, Y. (2007). Éducation et didactique: la tension essentielle. Éducation E Didactique, 1(1), 9-28.

Coldefy-Faucard, A. (2007). Le rire des Âmes Mortes: traduire Gogol. Geste, Traduire, (4), 87-93.

Conseil de la coopération culturelle, Comité de l'éducation, Division des langues vivantes, Strasbourg (2001). Cadre européen commun de référence pour les langues. Paris, France: Conseil de l'Europe / Didier. 
De Launay, M. (2014). La traduction: qu'est-ce que traduire?, France Culture, Les Nouveaux Chemins de la Connaissance, émission du 17 mars. Repéré à: http://www. franceculture.fr/emission-les-nouveaux-chemins-dela-connaissance-la-traduction-14-qu\%E2\%80\%99estce-que-traduire-2014-03-17

Descombes, V. (1996). Les institutions du sens. Paris, France: Minuit.

Descombes, V. (1998). La confusion des langues. Enquête, (6), 35-54.

De Pietro, J-F. (2013). Des approches plurielles, oui mais combien? Dans C. Troncy (dir.), Didactique du plurilinguisme. Approches plurielles des langues et des cultures. Autour de Michel Candelier (p. 227-238). Rennes, France: Presses universitaires de Rennes.

Dewey, J. (1938). Logique. Théorie de l'enquête. Paris, France: Presses universitaires de France.

Eco, U. (1993, novembre). Conférence d'ouverture. Communication présentée aux Assises de la Traduction Littéraire. Arles, France.

Eco, U. (2006). Dire presque la même chose. Expériences de traduction. Paris, France: Grasset.

Fleck, L. (1934 / 2005). Genèse et développement d'un fait scientifique. Paris, France: Les Belles Lettres.

Ginzburg, C. (1989). Mythes, emblèmes, traces: morphologie et histoire. Paris, France: Flammarion.

Geertz C. (1977). Interpretation of Cultures. New York, États-Unis: Basic Books.

Glock, H.-J. (2003). Dictionnaire Wittgenstein. Paris, France: Gallimard.

Gruson, B. (2006). L'enseignement d'une langue étrangère à l'école et au collège: vers une meilleure compréhension des situations didactiques mises en ouvre. Analyse comparative de l'action de deux professeurs de CM2 et de deux professeurs de sixième (Thèse, université Rennes 2 , Rennes, France).

Iser, W. (1976). L'acte de lecture. Théorie de l'effet esthétique. Paris, France: Mardaga.

Jakobson, R. (1959). On Linguistic Aspects of Translation. New York, États-Unis: Routledge.

Kervran, M. (2011). Le rôle de la mémoire didactique dans la mise en œuvre d'une didactique convergente du langage et des langues à l'école primaire. Revue Française de Pédagogie, (175), 89-98.

Kuhn, T. S. (1990). La tension essentielle: tradition et changement dans les sciences. Paris, France: Gallimard.

Kuhn, T. (2008). La structure des révolutions scientifiques. Paris, France: Flammarion.

Ladmiral, J.-R. (2010). Traduire: théorèmes pour la traduction. Paris, France: Gallimard.

Laugier, S. (2004). Duhem-Quine: de la sous-détermination de la théorie à l'indétermination de la traduction. Dans B. Cassin (dir.), Vocabulaire européen des philosophies, (p. 1318-1319). Paris, France: Éditions du Seuil.

Le Hénaff, C. (2013). L'anglais à l'école élémentaire: analyse didactique de l'articulation entre la langue et la culture (Thèse, université Rennes 2, Rennes, France).
Marzloff, M. (1999) Iser, compte rendu de lecture. Repéré sur le site de l'École Normale Supérieure de Lyon: http:// litterature.ens-lyon.fr//litterature/dossiers/theories-litteraires/reception/iserlyon.fr/litterature/dossiers/theories-litteraires/reception/iser

Mauss, M. (1950). Sociologie et anthropologie. Paris, France: Presses universitaires de France.

Michel, C. (2004). Manière, faire, style. Dans B. Cassin (dir.), Vocabulaire européen des philosophies, (p. 751755). Paris, France: Éditions du Seuil.

Ministère de l'Éducation nationale (2007). Programme de l'enseignement de langues vivantes étrangères au collège. Bulletin Officiel n ${ }^{\circ} 7 \mathrm{du}$ 26-04-07.

Momigliano, A. (1979). Sagesses barbares. Paris, France: Gallimard.

Moore, D. (2006). Plurilinguismes et école. Paris, France: Hatier.

Rastier, F. (2005). La traduction: interprétation et genèse du sens. Dans M. Lederer \& I. Fortunato (dir.), Le sens en traduction (p. 61-82). Paris, France: Minard.

Rivarol, A. (1784). De l'Universalité de la langue française. Repéré sur le site Histoire et Littérature: http://www. pourlhistoire.com/docu/discours.pdf

Ryle, G. (1971). Collected papers. Londres, Royaume-Uni : Hutchinson.

Sénat (2000). Article 211 du code pénal allemand. Repéré à : http://www.senat.fr/lc/lc21/lc211.html

Sensevy, G. (2011). Le Sens du Savoir. Éléments pour une théorie de l'action conjointe en didactique. Bruxelles, Belgique: De Boeck.

Steiner, G. (1998). Après Babel. Paris, France: Albin Michel.

Troncy, C. (dir.) (2013). Didactique du plurilinguisme. Approches plurielles des langues et des cultures. Autour de Michel Candelier. Rennes, France: Presses universitaires de Rennes.

Trouche, L. (2015). Enseigner les mathématiques à Shanghai. Repéré sur le site de la Commission Française pour l'Enseignement des Mathématiques: http://www. cfem.asso.fr/liaison-cfem/lettre-cfem-fevrier\%202015

Van Reeth, A. (2014). La traduction [interviews]. Dans A. Van Reeth (réalisatrice), Les Nouveaux Chemins de la Connaissance. Paris, France: France Culture.

Wittgenstein, L. (2004). Recherches philosophiques. Paris, France: Gallimard. 